\title{
Análisis del empleo de la metodología aprendizaje basado en proyectos como herramienta de desarrollo y evaluación de múltiples competencias transversales. Aplicación en grupos numerosos de asignaturas en la rama de ingeniería.
}

\author{
Y. Ye-Lin ${ }^{a}$, G. Prats-Boluda ${ }^{a}$, J. Garcia-Casado ${ }^{a}$, E. Guijarro Estelles ${ }^{\text {a }}$ y J.L. \\ Martinez-de-Juan ${ }^{\mathrm{a}}$ \\ a Universitat Politècnica de València. Departamento de Ingeniería Electrónica (yiye@eln.upv.es; \\ geprabo@eln.upv.es; jgarciac@eln.upv.es; eguijarro@eln.upv.es; jlmartinez@eln.upv.es).
}

\begin{abstract}
Previous experience has shown the feasibility of implementing active methodologies in small groups to work and evaluate cross skills. However, the assessment of cross skills in groups with a large number of students could lead to an excessive workload for teachers, questioning its sustainability. In the present work we propose the use of the project-based learning methodology and the portfolio to work and evaluate multiple skills in a core subject of 301 students, and report the experiences carried out. The results show that it is feasible to design and develop a formative activity to work and evaluate multiple skills in large groups without involving an excessive workload for students and teachers. In addition, $86.1 \%$ of the students positively valued the applied methodology that provides a more applied vision of the electronic instrumentation, favoring the acquisition of cross skills.
\end{abstract}

Keywords: projected based learning, comprehension and integration skill, practical thinking skill, problem solving skill, cross skills

\footnotetext{
Resumen

Experiencias previas han demostrado la viabilidad de implantar metodologías activas en grupos reducidos para trabajar y evaluar las competencias transversales. No obstante la evaluación de estas competencias en grupos numerosos puede suponer una carga de trabajo excesiva para el profesorado, cuestionando su sostenibilidad. En el presente trabajo se propone utilizar la metodología aprendizaje basado en proyectos y el portafolio para trabajar y evaluar múltiples competencias transversales en grupos numerosos. Concretamente se resumen las experiencias llevadas a cabo en una asignatura troncal de máster con 301 alumnos. Los resultados muestran que es factible diseñar y desarrollar una actividad formativa para trabajar y evaluar múltiples competencias en grupos numerosos sin suponer una carga de trabajo excesiva para el alumnado y el profesorado. Además el $86.1 \%$ de los alumnos valoraron positivamente la actividad manifestando que les proporciona una visión más aplicada de la instrumentación electrónica y favoreciendo la mejora en varias competencias transversales.
} 
Palabras clave: aprendizaje basado en proyectos, comprensión e integración, aplicación y pensamiento práctico, análisis y resolución de problemas, comunicación efectiva, competencias transversales

\section{Introducción}

La sociedad actual requiere de los egresados la formación en competencias transversales además de la formación en conocimientos genéricos y específicos de cada titulación. De hecho los empleadores muchas veces valoran las habilidades de empleabilidad transversales por encima de las habilidades específicas (Cotton, 2001). Con la implantación del Espacio Europeo de Educación Superior (EEES) y la entrada de los nuevos títulos aparece un cambio en el enfoque de la docencia desde la formación en contenidos a la formación en competencias. El enfoque del aprendizaje basado en competencias se fundamenta en la responsabilidad de aprender del estudiante y en el desarrollo de sus competencias iniciales a lo largo de su carrera. Esto supone que el aprendizaje no sólo se refiere al conocimiento nuevo que puede y debe adquirir el estudiante sino al desarrollo y evolución del modo de aprender, de aprender y mejorar cómo aplica los conocimientos a situaciones nuevas, cómo integra las actitudes y valores y los pone en juego, cómo incorpora las técnicas y métodos en su modo de actuar y afrontar las situaciones (Villa, 2011). Este nuevo enfoque requiere un aprendizaje significativo por parte del alumno que dote de sentido al material de aprendizaje mediante su interiorización. Esto también conlleva al cambio del modo de evaluación que no debe centrarse únicamente en la adquisición de conocimientos específicos. Como consecuencia de esta forma de evaluación, el examen, si bien continúa siendo un método de valoración de los contenidos asimilados, ha de combinarse con otras metodologías más adecuadas para determinar el nivel de dominio de las competencias. Esta circunstancia obliga al docente al uso de determinadas actividades académicas, que permitan una evaluación continua del estudiante a lo largo de todo el curso, y no sólo de una calificación a través de un examen final. En resumen, la adaptación al EEES ha supuesto una sustancial transformación de la docencia universitaria, tanto en lo referente a las metodologías empleadas, en las estructuras de las enseñanzas, o en la implantación de sistemas de evaluación y control de su calidad (Hermosilla, 2013).

En este contexto, la Universidad Politécnica de Valencia (UPV) ha puesto en marcha el proyecto de competencias transversales UPV, que tiene como objetivo principal acreditar las competencias transversales los estudiantes egresados en cualquiera de los títulos oficiales impartidos. Con el fin de unificar las competencias genéricas y analizando las clasificaciones de distintas agencias y organismos públicos de acreditación (EUR-ACE, $\mathrm{ABET}$, órdenes ministeriales $\mathrm{CIN}$, MECES, RD), se ha definido las competencias trasversales UPV (CT) que incluye un total de 13 competencias. Con la definición de las CT se pretende sintetizar el perfil competencial que adquieren los alumnos de la UPV. Partiendo de la hipótesis de que se trabajan las competencias transversales UPV en las distintas materias/asignaturas, a nivel institucional se realiza el seguimiento del progreso de los estudiantes a través de materias/asignaturas seleccionadas como puntos de control. En este contexto, la UPV contempla 3 niveles para cada CT: $1^{\circ}$ y $2^{\circ}$ de grado, $3^{\circ} \mathrm{y} 4^{\circ}$ de grado, y máster; que suponen diferentes niveles de dominio de las CT. Dentro de este marco,

(cc) EY-NC-ND 2017, Universitat Politècnica de València 
"Instrumentación y Control Industrial" es una asignatura troncal de primer curso del Máster Universitario en Ingeniería Industrial. Tiene cerca de 300 alumnos, y es punto de control de 4 competencias transversales que la entidad responsable de título recomienda evaluar. Éstas son: CT01 Comprensión e Integración; CT02 Aplicación y pensamiento práctico; CT03 Análisis y resolución de problemas; y CT08 Comunicación efectiva.

Existen diversas metodologías docentes que permiten a alumnos y docentes trabajar, desarrollar y evaluar el nivel de dominio de las distintas competencias, como el aprendizaje basado en proyectos y/o el uso del portafolio. El portafolio es una técnica de recopilación, compilación, colección y repertorio de evidencias y competencias profesionales que ha sido ampliamente utilizado como una herramienta de evaluación del desarrollo de competencias (Barragán, 2005; Villardón, 2006). El aprendizaje basado en proyectos se basa en el principio de usar problemas reales como punto de partida para la adquisición e integración de los nuevos conocimientos (Barrows, 1986). Es una metodología eficaz y flexible para ayudar al alumno a desarrollar y trabajar diversas competencias, i.e, análisis y resolución de problemas (habilidades de identificación de problemas relevantes del contexto profesional, búsqueda y manejo de información, etc..), integración de conocimientos multidisciplinares, aprendizaje autónomo y permanente, planificación de las estrategias que se van a utilizar para aprender, trabajo en equipo, pensamiento crítico, habilidades de manejar las herramientas, habilidades de comunicación (argumentación y representación de la información), habilidades de evaluación y autoevaluación (De Miguel, 2006, Prieto, 2006).

En cuanto a la implantación de nuevas metodologías docentes, experiencias piloto han demostrado la viabilidad de implantar y evaluar dichos métodos de enseñanza-aprendizaje en grupos reducidos (Prats-Boluda, 2016). Sin embargo puede suponer una dificultad adicional en grupos masivos y podría generar una excesiva carga de trabajo probablemente por encima de las posibilidades reales del profesorado (De la Peña, 2012; Grangel, 2013; Sánchez 2011), convirtiendo la actividad en insostenible de cara a cursos subsiguientes. A continuación se destacan las principales dificultades para el diseño de actividades para la evaluación de las competencias transversales en grupos numerosos (Sánchez, 2011):

- Los alumnos centran su esfuerzo en lo que se evalúa; el profesor concibe que es imposible abordar una evaluación continua, y que la única solución es el examen final

- La masificación es una característica de todo el centro y la cultura dominante todavía mantiene una tendencia clara a la importancia del examen final, predominando la calificación sobre la evaluación.

- Las resistencias al cambio pueden venir generadas de malas experiencias imbuidas por un diseño erróneo en las actividades propuestas.

- La falta de experiencia puede generar un grado de dificultad añadido. En muchas ocasiones, el hecho de no tener formación apropiada en la materia hace que el profesorado rehúya de ciertas actividades, limitándose a una evaluación superficial.

- El llevar a cabo un sistema de seguimiento del proceso de aprendizaje orientado a ir viendo cómo va aprendiendo el alumno, no siempre es posible debido a cuestiones de recursos. 
- Abordar una evaluación profunda del alumnado implica una dedicación mayor del profesorado, que en ocasiones puede desbordar las posibilidades reales del profesor.

En este trabajo se pretende diseñar una actividad formativa para trabajar y evaluar múltiples competencias transversales (CT01, CT02, CT03 y CT08 ) en grupos numerosos empleando la metodología aprendizaje basado en proyectos y haciendo uso del portafolio.

\section{Desarrollo de la innovación}

La experiencia que se presenta en el presente trabajo se ha desarrollado en el contexto de la asignatura troncal "Instrumentación y Control Industrial", ofertada en primer curso de la titulación Máster Universitario en Ingeniería Industrial, y específicamente en la parte relativa a la instrumentación electrónica. Se trata de una asignatura con un amplio temario que abarca desde la descripción de los principios básicos de los sensores, selección de los sensores apropiados para una aplicación concreta, instrumentación de las señales provenientes de los sensores, desarrollo del sistema de adquisición de datos, así como la estimación de los errores que aparecen en la cadena de medida. Como se indicó anteriormente, la asignatura tiene cerca de 300 alumnos matriculados cada curso, divididos en 6 grupos de teoría de alrededor de 50 alumnos.

Con el objetivo de trabajar y evaluar las múltiples competencias que son puntos de control en esta asignatura, se ha diseñado una actividad centrándose en el aprendizaje basado en proyectos y el uso del portafolio. En concreto, se han propuesto 8 retos abiertos que consisten en la monitorización de una magnitud física en un proceso industrial. En cada grupo de teoría se forman unos 12 equipos (entre 3-5 alumnos/equipo) para resolver 3 retos en el área de instrumentación electrónica. Los retos a resolver en cada grupo de teoría fueron seleccionados por el profesorado para que éstos estuvieran distribuidos lo más uniformemente posible entre los distintos grupos. El desarrollo del proyecto tiene un peso del $15 \%$ de la nota final de la asignatura y se divide en 3 fases que se describirán con detalle en las siguientes subsecciones y por tanto tendrá 3 actos de evaluación.

\subsection{Fase 1}

Durante esta fase, el profesor plantea en cada grupo de teoría 3 retos abiertos (de los 8 posibles) relativos a la monitorización de una cierta magnitud en un proceso industrial. En una primera aproximación se plantean de forma genérica e.g. la medida del volumen de un compuesto líquido en un depósito. No se aportan detalles específicos del caso planteado; en el ejemplo de un medidor de nivel no se detalla la composición o características del líquido, forma y dimensiones del depósito, condiciones ambientales, etc. El objetivo es que los alumnos, en el seno de cada equipo, propongan y valoren ideas lo más libremente posible para resolver el reto planteado, todo ello sin estar inicialmente condicionado por detalles específicos de cada aplicación.

Ante el reto planteado, cada equipo de trabajo dispone de 20 minutos para proponer distintas alternativas de posibles soluciones al reto planteado sin ningún tipo de restricción (sesión de Brainstorming). Es importante destacar que en primera actividad todo tipo de

(cc) EY-NC-ND 2017, Universitat Politècnica de València 
soluciones es susceptible de ser considerada. Al concluir dicho tiempo cada grupo entrega un listado al profesor con todas las alternativas propuestas, en principio cuantas más mejor.

Tras el primer acercamiento al problema y propuesta de ideas, cada equipo procede a valorar más en detalle la viabilidad y adecuación de las propuestas del listado anterior, a fin de reducir la lista de posibilidades a un máximo de 3. Cabe señalar que todavía no se conocen las condiciones específicas de aplicación por lo que estas soluciones deben cubrir un amplio espectro de posibles aplicaciones específicas (que será concretada en la siguiente fase). Los equipos disponen de 40 minutos para proporcionar un documento con las 3 soluciones posibles seleccionadas, indicando brevemente su principio de funcionamiento y sus potenciales ventajas y desventajas. La fase 1 es evaluada exclusivamente por el profesorado de cada grupo de teoría en base a la rúbrica de evaluación de la Fase 1 publicada previamente a la sesión de trabajo y que se adjunta en el anexo 1.

\subsection{Fase 2}

Una vez concluida la fase 1, el profesor proporciona a cada equipo los detalles de una aplicación concreta para cada uno de los retos planteados. En el ejemplo propuesto podrían ser detalles respecto a la composición del líquido, forma y dimensiones del depósito, condiciones específicas de la aplicación, etc. Con esta nueva información cada equipo debe elaborar su propuesta de solución definitiva. Para ello es necesario realizar un análisis crítico en mayor profundidad de las distintas alternativas preseleccionadas en la Fase 1.

Durante el desarrollo del trabajo, cada equipo debe preparar un portafolio que contenga: el plan de trabajo del equipo (PT) detallado, incluyendo la asignación de tareas, el diagrama de Gantt, y las actas de reuniones del equipo de trabajo; un documento explicativo de la estrategia de búsqueda de información (BI) empleada para la resolución del problema y la forma de valorar la calidad de la información encontrada; y por último la justificación de la toma de decisiones (TD) a lo largo del proyecto. Para ello, se les proporciona a los alumnos un documento donde se explica detalladamente en qué consiste el portafolio y los documentos que debe contener.

La evaluación del portafolio se realiza mediante la técnica de coevaluación. En cada grupo de teoría se formarán 9 (3 item x 3 retos) comités de "expertos" para cada ítem evaluable (documentos PT, BI y TD) y cada reto planteado en ese grupo. Cada comité de "expertos" de cada ítem evaluable (PT, BI y TD) estará formado por al menos un miembro de cada equipo siendo éste asignado libremente por cada equipo, es decir, la asignación de los roles alumnos expertos es una decisión de cada equipo y no del profesor. Los miembros de cada comité de expertos tienen asignado un reto a evaluar distinto al que debían desarrollar con sus equipos. En la figura 1 se muestra la formación de los comités evaluadores del ítem PT y la agrupación de los ítems evaluables del mismo tipo.

Como resultados de la evaluación del portafolio, cada comité evaluador rellena la rúbrica correspondiente de cada ítem (PT, BI y TD) de cada equipo. Al final de la sesión, los comités evaluadores se intercambia las rúbricas para generar una valoración global de cada equipo que contiene la evaluación de cada indicador de cada ítem y se proporciona el feedback a cada equipo. 


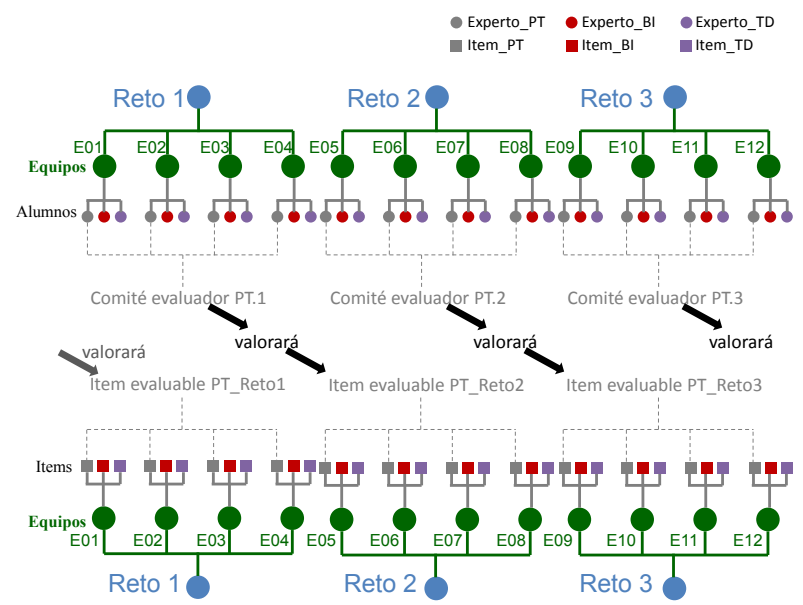

Figura 1: Esquema del método de coevaluación llevado a cabo para la evaluación del portafolio.

\subsection{Fase 3}

Una vez finalizada la sesión de evaluación del portafolio, cada equipo debe preparar una presentación con los detalles de la solución propuesta: tipo de sensor, principio de medida, montaje/colocación, posibles fuentes de error, limitaciones, principales ventajas e inconvenientes, valorando además aspectos como coste, mantenimiento, prestaciones de medida, etc. En los casos que sea posible se valora presentar sensores/sistemas industriales existentes en el mercado que puedan satisfacer las especificaciones del problema planteado de acuerdo a la solución propuesta por el grupo.

La presentación oral del trabajo se realiza en el aula de cada grupo de teoría con los medios disponibles, y tiene una duración máxima de 12 minutos para cada equipo. Tras la presentación de cada equipo se abre un turno abierto de preguntas y comentarios por parte del resto de equipos hacia el equipo que ha realizado la presentación (unos $3 \mathrm{~min}$ ).

Una vez concluidas las presentaciones de propuestas de solución de los grupos asociados a cada reto, el profesor evalúa la presentación del trabajo mediante la rúbrica de evaluación de la fase 3 (ver Anexo 1). Cada equipo valora qué solución le parece más adecuada de entre todas las presentadas tomando como referencia la rúbrica de evaluación de la fase 3 , entregando su valoración numérica global entre 0 y 10 al profesor. El resultado de dicha valoración por parte del alumnado se tiene en cuenta en la evaluación final de cada equipo.

En la tercera sesión de presentación que coincide con la última clase de la asignatura, se realiza una encuesta a los alumnos para conocer sus opiniones acerca del conjunto de actividades propuestas y determinar si el desarrollo del seminario ha permitido mejorar las competencias mediante un test auto-evaluación. Asimismo, también se pretende conocer el tiempo de dedicación del alumnado para desarrollar el proyecto. En el anexo 2 se adjunta la encuesta que se realizó al alumnado. 


\subsection{Evaluación de las competencias}

Con el objeto de evaluar las distintas competencias, se ha identificado la contribución de cada indicador de cada fase del proyecto a cada una de las competencias transversales (ver la última columna de las rúbricas adjuntas en el anexo I). Con ello para cada competencia a valorar se tiene el resultado de un determinado número de indicadores $\left(\mathrm{N}_{\mathrm{i}}\right)$ relativos a dicha competencia (CT01: 3 indicadores; СТ02: 8 indicadores; CT03: 10 indicadores; CT08: 6 indicadores). La evaluación final de cada competencia, así como de cada uno de sus indicadores, se corresponde con una escala categórica de 4 niveles: A Excelente/ejemplar; B Bien/Adecuado; C En desarrollo; D No alcanzado. La evaluación final de cada competencia a partir de las $\mathrm{N}_{\mathrm{i}}$ evaluaciones de cada indicador admite distintos enfoques. En un primer acercamiento, la evaluación final de cada competencia se corresponde con el resultado de evaluación más repetido entre sus indicadores asociados.

\section{Resultados}

En el proyecto participaron 288 alumnos matriculados en la asignatura. Se formaron inicialmente 70 equipos de trabajo de 3 a 5 alumnos. En la figura 2 se muestra la distribución de los equipos de trabajo en los 6 grupos de teoría. Todos los equipos han seguido la dinámica de actividad preprogramada, siendo la tasa de abandono de la actividad $0 \%$. Del total de alumnos matriculados, 237 alumnos han contestado la encuesta de autoevaluación en la última sesión de la presentación del trabajo.

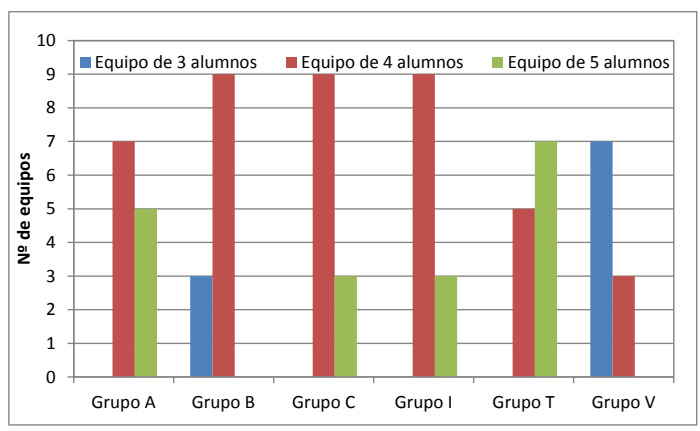

Figura 2: Distribución de los equipos de trabajo en función del número de alumnos por equipo en cada uno de los 6 grupos.

En la tabla 1 se detalla el tiempo de dedicación estimado del profesorado y del alumnado para el desarrollo del proyecto. Para el total de los alumnos encuestados, la dedicación promedio es de unas $17.3 \pm 8.1 \mathrm{~h}$, lo que se aproxima bastante a la estimación inicial del profesorado al diseñar la tarea $(13.8 \mathrm{~h} /$ alumno que corresponde a unos 0.7 créditos ECTS dedicado a esta parte de la asignatura). Por otro lado, la dedicación promedio del profesorado es de unos $26.6 \pm 9.8 \mathrm{~h}$, lo cual está dentro de lo que se puede considerar sostenible. Cabe señalar que el tiempo de dedicación del profesorado está principalmente destinado a la reunión grupal, y la preparación de los materiales docentes (preparación de los retos a resolver, definición y elaboración de las rúbricas, preparación del documento explicativo del proyecto y del portafolio). Parte de este trabajo en necesario únicamente en el primer curso de puesta en marcha de la acividad, que se ve amortizado en cursos subsiguientes. 
Tabla 1: Estimación del tiempo de dedicación al proyecto del profesorado y del alumnado.

\begin{tabular}{lccc}
\hline \multicolumn{1}{c}{ Tiempo de dedicación } & Individual (h) & Grupal (h) & Total (h) \\
\hline Alumnado & $7.5 \pm 5.0$ & $9.8 \pm 6.0$ & $17.3 \pm 8.1$ \\
Profesorado & $17.0 \pm 8.9$ & $9.6 \pm 1.5$ & $26.6 \pm 9.8$ \\
\hline
\end{tabular}

En la figura 3 se muestra la autoevaluación de los alumnos sobre sus competencias (CT01 Comprensión e Integración, CT02 Aplicación y pensamiento práctico, CT03 Análisis y resolución de problemas y CT08 Comunicación efectiva) antes y después de cursar la asignatura. Al inicio del curso, la gran mayoría de alumnos (entre $77.6 \%$ y $86.5 \%$ ) posee un nivel de dominio de las competencias trabajadas y evaluadas "Buena" y "Muy buena". En general, el número de alumnos cuyo nivel de dominio de las competencias se sitúa en el nivel "Muy bajo" y "Bajo" es reducido, lo cual es el resultado esperable dado que son alumnos de primero de máster y están cursando un nivel 3 de las competencias transversales de la UPV. Tras cursar la asignatura, se puede apreciar que en general el $\mathrm{n}^{\mathrm{o}} \mathrm{de}$ alumnos cuyo nivel de dominio de las competencias se sitúa en "Muy bajo", "Bajo" y "Buena" ha disminuido. El porcentaje de alumnos cuya competencia se sitúa en el nivel "Muy buena" y/o "Excelente" ha aumentado considerable (ver tabla 2) con incrementos de 24.9\% para el CT01, 28.3\% para el CT02 y CT03, 21.6\% para el CT08. Por otro lado, destacar que el porcentaje de alumnos con un nivel de competencia "Muy buena" y/o "Excelente" para CT01 y CT08 es considerablemente inferior que en el caso de las competencias CT02 y CT03. Este resultado fue sorprendente para los profesores, dado que la CT01 supuestamente es una de las competencias que se trabajan desde los primeros cursos del grado. En cambio, el resultado obtenido de la competencia CT08 muestra una carencia de formación en esta competencia en asignaturas de grado que puede ser debido a la dificultad asociada al trabajo de la misma en grupos numerosos.
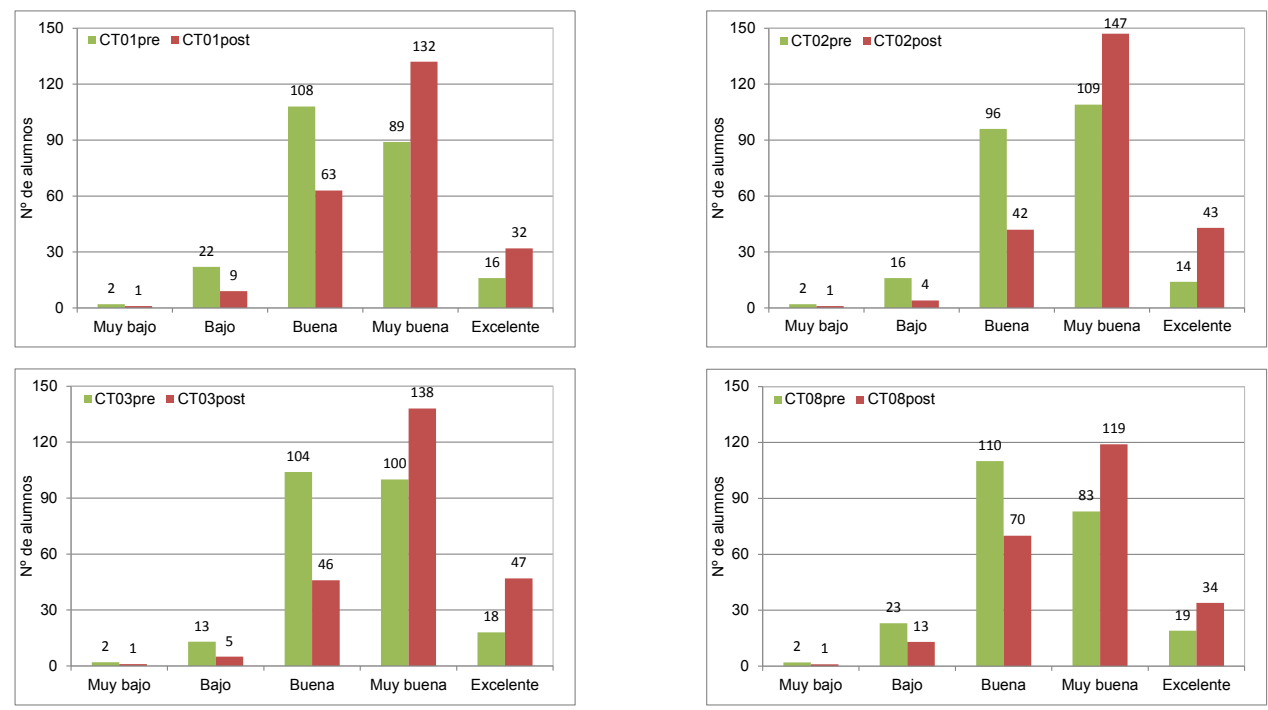

Figura 3: Resultados de la autoevaluación de los alumnos sobre el nivel de dominio de las competencias CT-01 (Comprensión e Integración), CT-02 (Aplicación y pensamiento práctico), CT-03 (Análisis y resolución de problemas), CT-08 (Comunicación efectiva) antes y después de cursar la asignatura. 
Tabla 2: Porcentaje de alumnos que posee un nivel de dominio de las competencias "Muy buena" o "Excelente" antes y después de cursar la asignatura.

\begin{tabular}{ccc}
\hline CT & Antes & Después \\
\hline CT01 & $44.3 \%$ & $69.2 \%$ \\
CT02 & $51.9 \%$ & $80.2 \%$ \\
CT03 & $49.8 \%$ & $78.1 \%$ \\
CT08 & $43.0 \%$ & $64.6 \%$
\end{tabular}

En la figura 4 se muestra la distribución de la evaluación de las 4 competencias objeto de estudio de acuerdo con el apartado 2.4. En general, la gran mayoría de alumnos (por encima del 95\%) posee un nivel de dominio de las competencias "Bien/Adecuado" y "Excelente". Se observa que el porcentaje de alumnos que han alcanzado un nivel de competencia "Excelente" es del orden 50\% o superior (СТ01: 50\%: СТ02: 64.2\%; СТ03 58.7\%; СТ08: $48.9 \%$ ). Estos resultados coinciden parcialmente con la auto-evaluación de los alumnos en la que se ponía de manifiesto que la competencia CT08 eran el punto débil de los alumnos, es decir, es la competencia más difícil de adquirir/desarrollar por parte del alumnado.

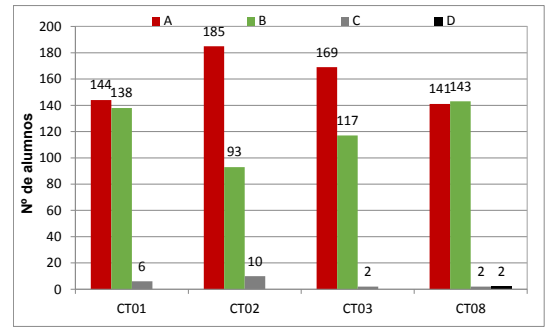

Figura 4: Distribución de la evaluación categórica de las competencias analizadas. A: Excelentelejemplar, B: Bien/adecuado, C: En desarrollo, D: No alcanzado

Además de las competencias puntos de control de la asignatura, un ratio importante de los alumnos consideran que el aprendizaje basado en proyectos ha permitido trabajar otras competencias (ver figura 5) como la "CT06 Trabajo en equipo y liderazgo" (70\%) y en menor medida "CT09 Pensamiento crítico" (54\%), "CT10 Planificación y gestión del tiempo" (48\%), "CT11 Aprendizaje permanente: Capacidad de auto-aprendizaje" (47\%) y "CT04 Innovación, creatividad y emprendimiento" (40\%). Destacar que también se obtuvieron indicadores de las competencias CT04 y CT09 en el desarrollo de las actividades propuestas y que también podrían ser de utilidad.

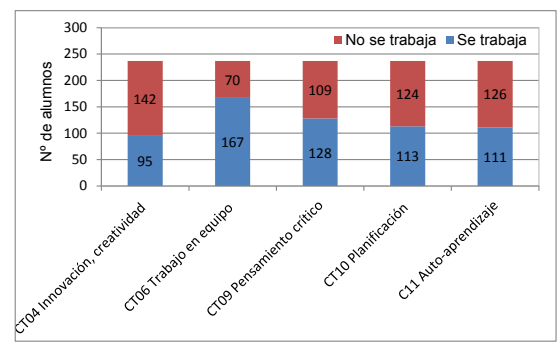

Figura 5: Número de alumnos que consideran que el desarrollo del proyecto han contribuido a la adquisición de otras competencias de las que la asignatura no es punto de control. 
En la figura 6 se muestra la valoración de los alumnos hacia el uso de metodología docente "aprendizaje basado en proyecto" en la asignatura Instrumentación y Control Industrial. En general, los alumnos han mostrado una actitud y una valoración muy positiva de la metodología docente aplicada. El $86.1 \%$ de alumnos valoran positivamente la metodología "aprendizaje basado en proyecto" e indican que les ha proporcionado una visión más aplicada de la instrumentación electrónica y favoreciendo a su vez la mejora en el nivel de dominio de diferentes competencias transversales. A pesar de ello existe un porcentaje reducido de alumnos (13.9\%) que mostró cierta resistencia al cambio en favor de las metodologías activas, arguyendo que las horas dedicadas a la actividad no compensan a los conocimientos adquiridos.

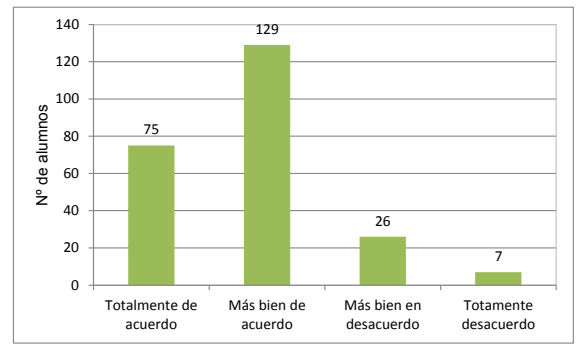

Figura 6: Valoración de los alumnos acerca del uso de la metodología docente "aprendizaje basado en proyecto" en la asignatura Instrumentación y Control industrial. Curso 16-17.

\section{Conclusión}

Se ha diseñado y llevado a cabo una actividad formativa para trabajar y evaluar las múltiples competencias transversales en grupos numerosos, empleando la metodología de aprendizaje basado en proyectos y el portafolio . El análisis de los resultados obtenidos indica que dicha actividad no supone una excesiva carga de trabajo ni para el alumnado ni para el profesorado. Tras finalizar el conjunto de actividades la consideración de los alumnos refleja un incremento en el número de alumnos que alcanzan dominio "muy bueno" o "excelente" de las competencias trasversales evaluadas (CT-01 Comprensión e Integración, CT-02 Aplicación y pensamiento práctico, CT-03 Análisis y resolución de problemas, CT-08 Comunicación efectiva). Los resultados de la evaluación del profesorado reflejan que la gran mayoría de alumnos (por encima del 95\%) posee un nivel de dominio de las competencias trabajadas y evaluadas de "Bien/Adecuado" y "Excelente". Además, tanto los alumnos $(86.1 \%)$ como el profesorado valoran positivamente la metodología aprendizaje basado en proyectos reflejando que proporciona una visión más aplicada de la instrumentación electrónica y favorece la adquisición de las competencias transversales.

\section{Agradecimientos}

Este trabajo está subvencionado parcialmente por la Escuela Técnica Superior de Ingenieros Industriales y el Vicerrectorado de Estudios, Calidad y Acreditación de la UPV (PIME B17, Convocatoria 2016-2017). 


\section{Referencias}

BARRAGAN SANCHEZ, R (2005) "El portafolio, metodología de evaluación y aprendizaje de cara al nuevo Espacio Europeo de Educación Superior. Una experiencia práctica en la Universidad de Sevilla." en Revista Latinoamericana de Tecnología Educativa, Vol 4, nº1, pp121-139.

BARROWS, H.S. (1986). "A Taxonomy of problema-based learning methods" en Medical Education, Vol 20 n6, pp481-486.

DE MIGUEL, M. (2006) "Metodologías de enseñanza para el desarrollo de competencias. Orientaciones para el profesorado universitario ante el Espacio Europeo de Educación Superior." en Educatio siglo XXI, Vol 24, pp 207-210.

COTTON K. (2001) "Developing Employability Skills" [online]. Available at: $<$ http://www.bikume.com/descargas/5/archivos/tesis-sobre-competencias-transversales-para-mejorarla-empleabilidaed.pdf $>$ [Consulta Junio 2016].

DE LA PEÑA AMORÓS M. (2012) "La evaluación de los alumnos a través del portafolio" en Revista Jurídica de Investigación e Innovación Educativa, Nº, pp81-94.

GRANGEL SEGUER R., CAMPOS SANCHO C. (2013) "Contratos de aprendizaje y evaluación entre iguales para responsabilizar al alumno de su aprendizaje" Actas de las XIX Jenui Castellón. pp 45-52.

HERMOSILlA Z., CLEMENTE M., TRINIDAD A., ANDRÉS J. (2013) “Competencia en comunicación oral: un reto para el ingeniero" En Garrigos et al. "New changes in technology and innovation." INNODOCT 2013. Universidad Politécnica de Valencia.

PRATS-BOLUDA G., YE-LIN Y., TRENÓR GOMIS B. (2016) “Análisis del uso del póster científico y de la revisión por pares como herramienta desarrollo de la competencia comunicación efectiva en estudiantes de grado en ingeniería" Congreso Nacional de Innovación Educativa y de Docencia en Red (IN-RED). Valencia.

PRIETO, L. (2006) "Aprendizaje activo en el aula universitario: el caso del aprendizaje basado en problemas, en Miscelánea Comillas." Revista de Ciencias Humanas y Sociales, Vol. 64. №124, pp 173-196.

SÁNCHEZ SANTAMARÍA J. (2011) "Evaluación de los aprendizajes universitarios: una comparación sobre sus posibilidades y limitaciones en el Espacio Europeo y Educación Superior" Revista de Formación e Innovación Educativa Universitaria, Vol $4 \mathrm{~N}^{\circ} 1$, pp 40-54

VILLA SÁNCHEZ A., POBLETE RUIZ M. (2011) "Evaluación de competencias genéricas: principios, oportunidades y limitaciones" Bordón, Vol 63 NoI, pp147-170.

VILLARDÓN GALLEGO M.L. (2006) "Evaluación del aprendizaje para promover el desarrollo de competencias" Educatio siglo XXI, Vol. 24, pp57-76. 
ANEXO I. Rúbricas de evaluación de la Fase 1

\begin{tabular}{|c|c|c|c|c|c|}
\hline \multirow{2}{*}{ Indicadores } & \multicolumn{4}{|c|}{ Descriptores } & \multirow{2}{*}{ Competencia } \\
\hline & D. No alcanzado & C. En desarrollo & B. Bien/adecuado & A. Excelente/ejemplar & \\
\hline $\begin{array}{c}\text { Aporta ideas y planteamiento } \\
\text { originales } \\
\text { (F1.1) }\end{array}$ & $\begin{array}{l}\text { No aporta ideas ni } \\
\text { planteamientos y muestra } \\
\text { una actitud cerrada y/o } \\
\text { pasiva antes las tareas }\end{array}$ & $\begin{array}{l}\text { Aporta alguna idea o } \\
\text { planteamiento ante } \\
\text { situaciones nuevas }\end{array}$ & $\begin{array}{c}\text { Aporta de forma detallada sus } \\
\text { propias ideas y/o planteamientos } \\
\text { originales }\end{array}$ & $\begin{array}{c}\text { Aporta una gran variedad de ideas y } \\
\text { planteamientos novedosos, con fluidez, } \\
\text { bien elaborados, aplicables a diferentes } \\
\text { casos }\end{array}$ & $\begin{array}{l}\text { CT-01 Comprensión e } \\
\text { Integración } \\
\text { CT-04 Innovación, } \\
\text { creatividad y } \\
\text { emprendimiento }\end{array}$ \\
\hline $\begin{array}{c}\text { Plasma de manera formal las } \\
\text { ideas } \\
\text { (F1.2) }\end{array}$ & $\begin{array}{l}\text { No plasma de manera } \\
\text { formal las ideas }\end{array}$ & $\begin{array}{c}\text { Plasma de manera limitada } \\
\text { las ideas que se han } \\
\text { generado }\end{array}$ & $\begin{array}{l}\text { Plasma de manera correcta las } \\
\text { ideas que se han generado }\end{array}$ & $\begin{array}{c}\text { Plasma de manera enriquecida las ideas } \\
\text { que se han generado }\end{array}$ & $\begin{array}{l}\text { CT-08 Comunicación } \\
\text { efectiva }\end{array}$ \\
\hline $\begin{array}{l}\text { Valora las implicaciones } \\
\text { prácticas (pros-contras) de } \\
\text { diversas alternativas o } \\
\text { soluciones (F1.3) }\end{array}$ & $\begin{array}{l}\text { No dispone de elementos de } \\
\text { juicio para identificar las } \\
\text { ventajas e inconvenientes de } \\
\text { diferentes soluciones }\end{array}$ & $\begin{array}{l}\text { Es capaz de comparar } \\
\text { diferentes soluciones, pero } \\
\text { no sabe valorar sus } \\
\text { implicaciones prácticas }\end{array}$ & $\begin{array}{l}\text { Identifica pros y contras de } \\
\text { diferentes soluciones y valora } \\
\text { sus implicaciones prácticas }\end{array}$ & $\begin{array}{l}\text { Reconsidera las alternativas en función de } \\
\text { la reflexión y valoración de las } \\
\text { implicaciones que tienen }\end{array}$ & $\begin{array}{l}\text { CT-02 Aplicación } \\
\text { pensamiento práctico } \\
\text { CT-03 Análisis y resolución } \\
\text { de problemas } \\
\text { CT-09 Pensamiento crítico }\end{array}$ \\
\hline
\end{tabular}

ANEXO I. Rúbrica de evaluación de la Fase 2: Plan de Trabajo

\begin{tabular}{|c|c|c|c|c|c|}
\hline \multirow{2}{*}{ Indicadores } & \multicolumn{4}{|c|}{ Descriptores } & \multirow{2}{*}{ Competencias } \\
\hline & D. No alcanzado & C. En desarrollo & B. Bien/adecuado & A. Excelente/ejemplar & \\
\hline $\begin{array}{c}\text { Identificación y Asignación de } \\
\text { las tareas } \\
\text { (PT.1) }\end{array}$ & $\begin{array}{l}\text { Distribución de las tareas } \\
\text { inapropiada con el número de } \\
\text { miembros del grupo. Las tareas } \\
\text { no se han identificado } \\
\text { correctamente }\end{array}$ & $\begin{array}{l}\text { Distribución de las tareas } \\
\text { inapropiada con el número de } \\
\text { miembros del grupo. No se } \\
\text { identifican todas las tareas }\end{array}$ & $\begin{array}{l}\text { Distribución de las tareas } \\
\text { adecuada con el número de } \\
\text { miembros del grupo. Definición } \\
\text { correcta. }\end{array}$ & $\begin{array}{l}\text { Distribución de las tareas } \\
\text { coherente con el número de } \\
\text { miembros del grupo. Definición } \\
\text { correcta y precisa. De las tareas }\end{array}$ & $\begin{array}{c}\text { CT-01 Comprensión e } \\
\text { Integración } \\
\text { CT-03 Análisis y resolución de } \\
\text { problemas }\end{array}$ \\
\hline $\begin{array}{l}\text { Diagrama de Gantt } \\
\text { (PT.2) }\end{array}$ & $\begin{array}{l}\text { Temporización inadecuada de la } \\
\text { programación de las tareas y/o } \\
\text { Formato de presentación pobre }\end{array}$ & $\begin{array}{l}\text { Temporización poco adecuada } \\
\text { de la programación de las tareas } \\
y / o \\
\text { Formato de presentación regular }\end{array}$ & $\begin{array}{l}\text { Temporización adecuada de la } \\
\text { programación de las tareas y/o } \\
\text { Formato de presentación bueno }\end{array}$ & $\begin{array}{l}\text { Temporización adecuada de la } \\
\text { programación de las tareas y/o } \\
\text { Formato de presentación } \\
\text { excelente }\end{array}$ & $\begin{array}{l}\text { CT-03 Análisis y resolución de } \\
\text { problemas }\end{array}$ \\
\hline $\begin{array}{l}\text { Actas de reunión } \\
\text { (PT.3) }\end{array}$ & $\begin{array}{l}\text { Incompletas: la recopilación de } \\
\text { la información es muy } \\
\text { deficiente en cuanto al } \\
\text { contenido tratado y el informe } \\
\text { elaborado }\end{array}$ & $\begin{array}{l}\text { Parcialmente incompleta: No } \\
\text { recopila todos los puntos a } \\
\text { tratar. Los informes están poco } \\
\text { elaborados y/o con } \\
\text { incorrecciones. }\end{array}$ & $\begin{array}{l}\text { Completa: recopila todos los } \\
\text { puntos tratados. Los informes } \\
\text { son claros, concisos y con } \\
\text { leguaje adecuado. }\end{array}$ & $\begin{array}{l}\text { Exhaustivas: recopila todos y } \\
\text { cada uno de los puntos tratados. } \\
\text { Los informes son muy claros, } \\
\text { concisos y excelentes en la } \\
\text { corrección léxica y gramatical } \\
\text { empleando el lenguaje adecuado }\end{array}$ & CT-08 Comunicación efectiva \\
\hline
\end{tabular}


ANEXO I. Rúbrica de evaluación de la Fase 2: Búsqueda de Información

\begin{tabular}{|c|c|c|c|c|c|}
\hline \multirow{2}{*}{ Indicadores } & \multicolumn{4}{|c|}{ Descriptores } & \multirow{2}{*}{ Competencias } \\
\hline & D. No alcanzado & C. En desarrollo & B. Bien/adecuado & A. Excelente/ejemplar & \\
\hline $\begin{array}{l}\text { Definición de } \\
\text { conceptos y uso de } \\
\text { palabras clave } \\
\text { adecuadas } \\
\text { (BI.1) }\end{array}$ & $\begin{array}{l}\text { Se escasa o incorrecta definición de los } \\
\text { conceptos asociados a la resolución del } \\
\text { problema planteado y/o deficiente o } \\
\text { incorrecta definición de las palabras } \\
\text { clave. No se combinan de forma } \\
\text { adecuada }\end{array}$ & $\begin{array}{c}\text { No se han identificado en su } \\
\text { totalidad los conceptos } \\
\text { asociados a la resolución del } \\
\text { problema planteado. } \\
\text { Falta de concreción en } \\
\text { definición de las palabras clave. } \\
\text { Escasez de palabras clave o no } \\
\text { se combinan de la forma más } \\
\text { adecuada } \\
\end{array}$ & $\begin{array}{l}\text { Se han identificado en su mayoría } \\
\text { los conceptos asociados a la } \\
\text { resolución del problema } \\
\text { planteado. } \\
\text { Buena definición de las palabras } \\
\text { clave aunque puede que no } \\
\text { cubran la totalidad de los } \\
\text { aspectos a tratar. Se combinan de } \\
\text { forma adecuada. }\end{array}$ & $\begin{array}{l}\text { Se han identificado perfectamente los } \\
\text { conceptos asociados a la resolución del } \\
\text { problema planteado. Excelente definición de } \\
\text { las palabras clave. Se combinan de forma } \\
\text { adecuada. }\end{array}$ & $\begin{array}{c}\text { CT-01 } \\
\text { Comprensión e } \\
\text { integración } \\
\text { CT-02 Aplicación } \\
\text { y pensamiento } \\
\text { práctico } \\
\text { CT-03 Análisis y } \\
\text { resolución de } \\
\text { problemas } \\
\end{array}$ \\
\hline $\begin{array}{l}\text { Correcta selección de } \\
\text { los resultados } \\
\text { relevantes (BI.2) }\end{array}$ & $\begin{array}{l}\text { Fuentes poco o nada contrastadas } \\
\text { (artículos de opinión de autores sin } \\
\text { reconocido prestigio, páginas web no } \\
\text { oficiales) Y/O } \\
\text { Resultados insuficientes para poder } \\
\text { resolver el problema planteado }\end{array}$ & $\begin{array}{l}\text { La mayoría de las fuentes no } \\
\text { están contrastadas } Y / O \\
\text { Resultados incompletos } \\
\text { permiten resolver parciamente } \\
\text { el problema }\end{array}$ & $\begin{array}{l}\text { Incluye Fuentes contrastadas } \\
\text { pero algunas no lo son tanto. } \\
\text { Y/O } \\
\text { Resultados completos para poder } \\
\text { resolver el problema }\end{array}$ & $\begin{array}{l}\text { Fuentes contrastadas (fabricantes, } \\
\text { distribuidores, autores, de reconocido } \\
\text { prestigio) Y/O Resultados exhaustivos para } \\
\text { poder seleccionar de forma correcta la } \\
\text { solución óptima al problema }\end{array}$ & $\begin{array}{l}\text { CT-02 Aplicación } \\
\text { y pensamiento } \\
\text { práctico } \\
\text { CT-03 Análisis y } \\
\text { resolución de } \\
\text { problemas }\end{array}$ \\
\hline $\begin{array}{l}\text { Documentación } \\
\text { adecuada de los } \\
\text { resultados (BI.3) }\end{array}$ & $\begin{array}{l}\text { El documento está muy poco elaborado, } \\
\text { incompleto y/o la selección del } \\
\text { contenido no es adecuada (contenido } \\
\text { irrelevante o falta contenido) }\end{array}$ & $\begin{array}{l}\text { El documento poco elaborado, } \\
\text { una parte importante del } \\
\text { contenido no ha sido } \\
\text { seleccionado de forma } \\
\text { adecuada (irrelevante, } \\
\text { redundante) } \\
\end{array}$ & $\begin{array}{l}\text { El documento es completo con } \\
\text { una buena selección del } \\
\text { contenido aunque puede contener } \\
\text { alguna información poco } \\
\text { relevante. }\end{array}$ & $\begin{array}{l}\text { El documento es completo y se detallan de } \\
\text { forma clara los resultados de la búsqueda } \\
\text { con una excelente selección del contenido } \\
\text { (no contenido irrelevante) }\end{array}$ & $\begin{array}{l}\text { CT-08 } \\
\text { Comunicación } \\
\text { efectiva }\end{array}$ \\
\hline
\end{tabular}

\section{ANEXO I. Rúbrica de evaluación de la Fase 2: Toma de Decisión}

\begin{tabular}{|c|c|c|c|c|c|}
\hline \multirow{2}{*}{ Indicadores } & \multicolumn{4}{|c|}{ Descriptores } & \multirow{2}{*}{ Competencias } \\
\hline & D. No alcanzado & C. En desarrollo & B. Bien/adecuado & A. Excelente/ejemplar & \\
\hline $\begin{array}{l}\text { Alternativas propuestas } \\
\text { como solución al } \\
\text { problema (TD.1) }\end{array}$ & $\begin{array}{l}\text { Muy pocas alternativas de } \\
\text { solución }\end{array}$ & $\begin{array}{l}\text { Se echa en falta alguna } \\
\text { alternativas de solución }\end{array}$ & $\begin{array}{l}\text { Suficientemente representativas } \\
\text { alternativas de solución }\end{array}$ & $\begin{array}{l}\text { Gran abanico de alternativas de } \\
\text { solución }\end{array}$ & $\begin{array}{l}\text { CT-02 Aplicación y pensamiento } \\
\text { práctico } \\
\text { CT-03 Análisis y resolución de } \\
\text { problemas } \\
\end{array}$ \\
\hline $\begin{array}{l}\text { Criterios de valoración de } \\
\text { las alternativas (TD.2) }\end{array}$ & $\begin{array}{l}\text { Inadecuados para resolver el } \\
\text { problema y/o } \\
\text { escasos, no cubren todos los } \\
\text { aspectos del problema }\end{array}$ & $\begin{array}{c}\text { En general adecuados pero } \\
\text { quedan aspectos por definir para } \\
\text { la correcta resolución del } \\
\text { problema }\end{array}$ & $\begin{array}{c}\text { Adecuados, } \\
\text { cubren la gran mayoría aspectos } \\
\text { para resolver del problema }\end{array}$ & $\begin{array}{l}\text { Perfectamente definidos para } \\
\text { resolver el problema } \\
\text { Cubren todos los aspectos del } \\
\text { problema }\end{array}$ & $\begin{array}{l}\text { CT-02 Aplicación y pensamiento } \\
\text { práctico } \\
\text { CT-03 Análisis y resolución de } \\
\text { problemas } \\
\end{array}$ \\
\hline
\end{tabular}

(c) BY-NC-ND 2017, Universitat Politècnica de València 


\section{ANEXO I. Rúbrica de evaluación de la Fase 3: Presentación Oral del trabajo}

\begin{tabular}{|c|c|c|c|c|c|}
\hline \multirow{2}{*}{ Indicadores } & \multicolumn{4}{|c|}{ Descriptores } & \multirow{2}{*}{ Competencias } \\
\hline & D. No alcanzado & C. En desarrollo & B. Bien/adecuado & A. Excelente/ejemplar & \\
\hline $\begin{array}{l}\text { La exposición está debidamente } \\
\text { preparada y se ajusta al tiempo } \\
\text { establecido (F3.1) }\end{array}$ & $\begin{array}{c}\text { La exposición no está } \\
\text { debidamente estructurada y/o } \\
\text { no se ajusta al tiempo } \\
\text { establecido }\end{array}$ & $\begin{array}{l}\text { La exposición está organizada, } \\
\text { pero hay demasiada o escasa } \\
\text { información y/o no se ajusta al } \\
\text { tiempo establecido }\end{array}$ & $\begin{array}{l}\text { La audiencia puede captar } \\
\text { claramente la eficaz estructura } \\
\text { del contenido y la exposición se } \\
\text { ajusta al tiempo establecido }\end{array}$ & $\begin{array}{l}\text { La selección y organización del } \\
\text { contenido y su ilustración } \\
\text { (ejemplo, analogías...) son } \\
\text { coherentes y la exposición se } \\
\text { ajusta al tiempo establecido }\end{array}$ & CT-08 Comunicación efectiva \\
\hline $\begin{array}{l}\text { Respeta las normas lingüísticas } \\
\text { (corrección) y se expresa de } \\
\text { manera correcta (claridad). La } \\
\text { comunicación no verbal es } \\
\text { apropiada (F3.2) }\end{array}$ & $\begin{array}{c}\text { No realiza un buen uso del } \\
\text { lenguaje y/o no utiliza la } \\
\text { terminología específica de la } \\
\text { materia } \\
\text { Su lenguaje no verbal no } \\
\text { respalda y/o distrae su discurso } \\
\text { verbal }\end{array}$ & $\begin{array}{l}\text { La presentación no es fluida } \\
\text { (lenguaje limitado) y utiliza } \\
\text { escasa terminología específica o } \\
\text { lo hace inapropiadamente } \\
\text { Su lenguaje no verbal muestra } \\
\text { alguna/s deficiencia/s }\end{array}$ & $\begin{array}{l}\text { Respeta las normas lingüísticas } \\
\text { y se expresa de manera correcta, } \\
\text { utilizando de modo pertinente la } \\
\text { terminología específica } \\
\text { Su lenguaje no verbal es } \\
\text { coherente con el verbal }\end{array}$ & $\begin{array}{l}\text { La exposición se adapta a la } \\
\text { audiencia, haciendo uso de un } \\
\text { lenguaje y de una terminología } \\
\text { específicos, apropiados, } \\
\text { precisos y ricos } \\
\text { Su lenguaje no verbal refuerza } \\
\text { el verbal: gestos firmes, ritmo } \\
\text { variado y entonación } \\
\text { intencional }\end{array}$ & CT-08 Comunicación efectiva \\
\hline $\begin{array}{l}\text { Refuerza sus ideas con la ayuda } \\
\text { de los medios de apoyo (F3.3) }\end{array}$ & $\begin{array}{l}\text { No utiliza los medios de apoyo } \\
\text { apropiados o hace de un mal } \\
\text { uso de los mismos }\end{array}$ & $\begin{array}{l}\text { No siempre utiliza los medios } \\
\text { de apoyo más apropiados o no } \\
\text { hace el mejor uso de los mismos }\end{array}$ & $\begin{array}{l}\text { Los medios de apoyo } \\
\text { contribuyen a que el discurso se } \\
\text { comprenda mejor, enfatizando } \\
\text { sus puntos clave }\end{array}$ & $\begin{array}{l}\text { Los medios de apoyo destacan } \\
\text { por su calidad/originalidad y } \\
\text { contribuyen a que el discurso se } \\
\text { comprenda mejor }\end{array}$ & CT-08 Comunicación efectiva \\
\hline $\begin{array}{l}\text { Búsqueda de alternativas y } \\
\text { criterios de valoración de las } \\
\text { alternativas (F3.4) }\end{array}$ & $\begin{array}{l}\text { Inadecuados para resolver el } \\
\text { problema y/o escasos, no } \\
\text { cubren todos los aspectos del } \\
\text { problema }\end{array}$ & $\begin{array}{l}\text { En general adecuados pero } \\
\text { quedan aspectos por definir para } \\
\text { la correcta resolución del } \\
\text { problema }\end{array}$ & $\begin{array}{c}\text { Adecuados, cubren la gran } \\
\text { mayoría aspectos para resolver } \\
\text { el problema }\end{array}$ & $\begin{array}{l}\text { Perfectamente definidos para } \\
\text { resolver el problema. Cubren } \\
\text { todos los aspectos del problema }\end{array}$ & $\begin{array}{c}\text { CT-02 Aplicación y } \\
\text { pensamiento práctico } \\
\text { CT-03 Análisis y resolución de } \\
\text { problemas }\end{array}$ \\
\hline $\begin{array}{l}\text { Selección y desarrollo de la } \\
\text { solución (F3.5) }\end{array}$ & $\begin{array}{l}\text { Deficiente comparativa entre las } \\
\text { diferentes alternativas } \\
\text { propuestas. La justificación de } \\
\text { la solución muy poco elaborada }\end{array}$ & $\begin{array}{l}\text { Coherente con los criterios de } \\
\text { selección. La comparativa entre } \\
\text { las alternativas propuestas es } \\
\text { incompleta. Justificación pobre } \\
\text { de la solución }\end{array}$ & $\begin{array}{l}\text { Es coherente con los criterios de } \\
\text { selección. La comparativa entre } \\
\text { las diferentes alternativas } \\
\text { propuestas completa. } \\
\text { Justificación adecuada. }\end{array}$ & $\begin{array}{c}\text { Es coherente con los criterios de } \\
\text { valoración. Excelente } \\
\text { comparativa entre las } \\
\text { alternativas y justificación } \\
\text { exhaustiva de la solución } \\
\text { adoptada }\end{array}$ & $\begin{array}{c}\text { CT-02 Aplicación y } \\
\text { pensamiento práctico } \\
\text { CT-03 Análisis y resolución de } \\
\text { problemas }\end{array}$ \\
\hline
\end{tabular}




\section{ANEXO II. Encuesta sobre el seminario de instrumentación Curso 16-17}

1.- Indique el $\mathrm{n}^{\mathrm{o}}$ de horas de trabajo ha dedicado a nivel individual (fuera del horario de clase y sin contar las reuniones grupales) en el proyecto: búsqueda de información, elaboración del portafolio y la presentación, etc. Respuesta: horas

2.- Indique el $\mathrm{n}^{\circ}$ de horas ha dedicado a nivel grupal en el desarrollo del proyecto (fuera del horario de clase y sólo las reuniones grupales). Respuesta: horas

3.- Realice una autoevaluación de las siguientes competencias antes de comenzar el curso:

CT01 Comprensión e Integración $\quad \square$ Muy bajo $\square$ Bajo $\square$ Buena $\square$ Muy buena $\square$ Excelente
CT02 Aplicación y pensamiento práctico $\square$ Muy bajo $\square$ Bajo $\square$ Buena $\square$ Muy buena $\square$ Excelente
CT03 Análisis y resolución de problemas $\square$ Muy bajo $\square$ Bajo $\square$ Buena $\square$ Muy buena $\square$ Excelente
CT08 Comunicación efectiva $\quad \square$ Muy bajo $\square$ Bajo $\square$ Buena $\square$ Muy buena $\square$ Excelente

4.- Realice una autoevaluación de las siguientes competencias después de cursar la asignatura:

CT01 Comprensión e Integración $\quad \square$ Muy bajo $\square$ Bajo $\square$ Buena $\square$ Muy buena $\square$ Excelente
CT02 Aplicación y pensamiento práctico $\square$ Muy bajo $\square$ Bajo $\square$ Buena $\square$ Muy buena $\square$ Excelente
CT03 Análisis y resolución de problemas $\square$ Muy bajo $\square$ Bajo $\square$ Buena $\square$ Muy buena $\square$ Excelente
CT08 Comunicación efectiva $\quad \square$ Muy bajo $\square$ Bajo $\square$ Buena $\square$ Muy buena $\square$ Excelente

5.- Además de las competencias anteriores, el desarrollo del proyecto le ha ayudado a mejorar en las siguientes competencias (multi-respuesta):

$\square$ Capacidad auto-aprendizaje para emprender estudios posteriores con alto grado de autonomía

$\square$ Trabajo en equipo y liderazgo

$\square$ Planificación y gestión del tiempo

$\square$ Innovación, creatividad y emprendimiento

$\square$ Pensamiento crítico

6.- ¿Considera que el aprendizaje basado en proyecto es una buena metodología docente que favorece una visión más aplicada de la instrumentación electrónica y el desarrollo de las competencias transversales y por tanto deberá seguir empleándose en la asignatura?

$\square$ Totalmente de acuerdo

$\square$ Más bien en desacuerdo $\square$ Más bien de acuerdo

$\square$ Totalmente en desacuerdo 